\title{
Behavioral contrast as a function of component duration for leverpressing using a within-session procedure
}

\author{
FRANCES K. MCSWEENEY and CAM L. MELVILLE \\ Washington State University, Pullman, Washington
}

\begin{abstract}
Ten rats pressed levers for food reinforcers delivered by multiple schedules. Behavioral contrast was measured using a within-session procedure that presented the baseline and contrast schedules within single sessions. The absolute sizes of both positive and negative contrast increased and then decreased as components lengthened. Negative induction occurred when components were very short. These results question theories that predict that the size of contrast will vary inversely with component duration. They support theories that attribute positive and negative contrast to similar theoretical mechanisms. A comparison of the present results with those of past studies indicates that keypecking by pigeons and leverpressing by rats change as different functions of component duration. Treadlepressing by pigeons and leverpressing by rats change as similar functions. These findings challenge general process theories that argue that all responses obey the same behavioral laws.
\end{abstract}

An important question in the area of conditioning is whether or not all responses obey the same behavioral laws (e.g., Seligman, 1970). This question has been addressed in the area of multiple-schedule behavioral contrast. Behavioral contrast is an inverse relation between the rate of responding during a constant component of a multiple schedule and the conditions of reinforcement in the other component. Positive contrast is an increase in responding during a constant component, with a worsening of reinforcement conditions in the other component. Negative contrast is a decrease in responding, with improvements in the other component.

Most theories of contrast are general theories that attribute contrast to similar processes for all responses (e.g., Herrnstein, 1970; Hinson \& Staddon, 1978; McSweeney, 1987; Williams, 1983). Only the additive theories (e.g., Gamzu \& Schwartz, 1973; Hearst \& Jenkins, 1974; Rachlin, 1973) argue that different factors are involved. They argue that the stimulus-reinforcer relation contributes to contrast for some responses (e.g., keypecking), but not for others (e.g., treadlepressing or leverpressing).

Early studies weakly questioned the general theories. These studies failed to find positive contrast when pigeons pressed treadles under conditions that produced it when pigeons pecked keys, seemingly rejecting general theories (Hemmes, 1973; McSweeney, 1978; Westbrook, 1973). But failures to find treadlepress contrast might be attributed to methodological problems rather than to fundamental differences between the responses. Such

This research was partially supported by NIMH Grant MH42466. Reprint requests should be sent to Frances K. McSweeney, Department of Psychology, Washington State University, Pullman, WA 99164-4820. methodological problems include difficulty detecting changes in the generally low rates of treadlepressing (e.g., Davison \& Ferguson, 1978), poor discrimination between the components, or an insensitivity of pressing to reinforcement (e.g., Staddon, 1982).

More recent data provide a stronger challenge. They show that contrast generally varies inversely with component duration when pigeons peck keys (McSweeney, 1982) and directly with component duration when pigeons press treadles (McSweeney, 1982; McSweeney, Dougan, Higa, \& Farmer, 1986). These data are stronger because they cannot easily be attributed to methodological problems. Methodological problems might explain why contrast fails to occur for a response, but they cannot explain why contrast changes as different functions of component duration for different responses. Finding functional differences suggests that the responses differ in fundamental ways, more seriously questioning the general theories.

The present study provides additional information about functional similarities and differences among responses. It examines the size of positive and negative behavioral contrast as a function of component duration when rats press levers for food reinforcers. The results are then compared with the results of similar experiments concerning keypecking and treadlepressing of pigeons.

The present study also provides evidence about the symmetry of positive and negative contrast. Some theories predict that positive and negative contrast will vary as similar functions of the same variables (e.g. , Hermstein, 1970; Hinson \& Staddon, 1978; Rachlin, 1973); others argue that they need not do so (Schwartz, 1975). Past data indicate that positive and negative contrast do vary similarly. Both types of contrast generally decrease with increases in component duration when pigeons peck keys 
(McSweeney, 1982). Both types generally increase with increases in component duration when pigeons press treadles (McSweeney et al., 1986). Both types also increase with increases in baseline rate of reinforcement when pigeons peck keys (McSweeney et al., 1986). The present study will provide an additional test of theories that predict symmetry.

In the present experiment, a within-session method of measuring contrast was used. Conventional, acrosssessions, procedures modify reinforcement frequency in one component across successive phases from baseline to contrast and then back to baseline (e.g., McSweeney et al., 1986). The present, within-session, procedure measures contrast and baseline within single sessions. Withinsession procedures have been used occasionally in the past by other investigators (e.g., Coelho de Rose, 1986; Williams, 1979), but most studies of contrast use acrosssessions procedures.

The within-session procedure was developed to deal with two problems that have discouraged functional studies of contrast in the past. First, the across-sessions procedure requires many sessions. Each measurement of contrast requires exposure to a multiple schedule in each of three phases, with each phase lasting 30 to 40 sessions. Second, fluctuations in responding that occur over these long periods confound the measurement of contrast. Rates of responding may double from one baseline schedule to its recovery (e.g., McSweeney et al., 1986; Spealman \& Gollub, 1974), severely limiting the accuracy of quantitative statements about the size of contrast.

The within-session procedure addresses these problems. It reduces the amount of time required to measure contrast, as well as the fluctuations in responding that occur over time, because it measures baseline and contrast within single sessions. It also produces results similar to those produced by the across-sessions procedure (McSweeney \& Melville, 1988).

\section{METHOD}

\section{Subjects}

Ten naive rats bred from Sprague-Dawley stock served as subjects. They were approximately 120 days old at the start of the experiment and were maintained at approximately $90 \%$ of their freefeeding body weights. Five subjects were exposed to a procedure designed to produce positive contrast; the remaining 5 subjects were exposed to a procedure designed to produce negative contrast.

\section{Apparatus}

The apparatus was a Gerbrands operant conditioning unit for rats $(20.5 \times 19.5 \times 23.5 \mathrm{~cm})$. Two $5 \times 1 \mathrm{~cm}$ levers extended $1.5 \mathrm{~cm}$ into the chamber. They were located $10 \mathrm{~cm}$ from the ceiling and $1.5 \mathrm{~cm}$ from each side of the apparatus. The levers were connected to microswitches that required approximately $45 \mathrm{~g}$ to operate. Two lights ( $1 \mathrm{~cm}$ in diameter) were located above each lever, $3.5 \mathrm{~cm}$ below the ceiling. One light was $1.5 \mathrm{~cm}$ from the side wall; the other was $3 \mathrm{~cm}$ medial to the first. The lights over the left lever were white; those over the right lever were red. The houselight consisted of two clear lights $(1 \mathrm{~cm}$ in diameter) located in the ceil- ing near the back wall. Access to food was through a 4.5-cm square hole, centered between the sides and $1 \mathrm{~cm}$ from the floor. One 45$\mathrm{mg}$ Noyes pellet served as a food reinforcer.

The experimental enclosure was housed in a sound-attenuating chamber. A ventilating fan masked noises from outside the chamber. Experimental events were programmed by a SYM microcomputer located in another room.

\section{Procedure}

The subjects were trained to press both levers through a shaping by successive approximations procedure. They were then placed on a multiple schedule that delivered high rates of food reinforcement in the two components. Rates of reinforcement were gradually reduced until the subjects responded on a multiple variableinterval 15-sec variable interval 15-sec (multiple VI 15-sec VI 15sec) schedule.

Once the experiment began, each experimental session was divided into two halves that were conducted successively. During the first (baseline) half, a multiple VI 15-sec VI 15-sec schedule was presented to all subjects. Reinforcers for one component were obtained by pressing the left lever; reinforcers for the other component were obtained by pressing the right lever. High rates of reinforcement were used because leverpress contrast is not always found when lower rates are used (Dougan, McSweeney, \& Farmer, 1985).

During the second (contrast) half of each session, a multiple VI 15-sec extinction schedule was presented to the 5 subjects exposed to a positive contrast procedure. A multiple VI 15-sec VI 7-sec schedule was presented to the 5 subjects exposed to a negative contrast procedure. The VI 15-sec component was arranged on the left lever. Extinction or the VI 7-sec schedule was arranged on the right lever.

The lights above the appropriate lever were illuminated when its component was available, and the components were alternated. Component durations varied across experimental conditions. The following durations were presented to all subjects in the following order: $60 \mathrm{sec}, 30 \mathrm{sec}, 16 \mathrm{~min}, 5 \mathrm{sec}$, and $3 \mathrm{~min}$. The number of components presented per session varied with component duration to prevent some sessions from becoming extremely long. The following number of components were presented in each of the baseline and contrast parts of the session for the $60-\mathrm{sec}, 30-\mathrm{sec}, 16-$ $\min , 5-\mathrm{sec}$, and 3-min components, respectively: $20,40,2,240$, and 8.

In all conditions, a 3-sec timeout separated the components. During the timeout, the lights over the levers were not illuminated and no reinforcers were delivered. The timeout was included because the studies with which the present one will be compared included a timeout. The timeout also ensured that any time required to move from one operandum to the other would not be included in calculating the size of contrast. If it were, it would distort the measures more for shorter components than for longer components, making the results difficult to interpret.

All reinforcers were scheduled according to a 25 -interval series constructed according to the procedure outlined in Fleshler and Hoffman (1962). Reinforcers that were scheduled but not collected before a component changed were held over for the next presentation of that component. Sessions were conducted daily, five to six times per week. The first experimental condition was conducted to stability (67 sessions) to accustom subjects to the procedure. Responding was considered to be stable when the response rates during the last five sessions fell with the range of rates during all of the earlier sessions. All other conditions were conducted for 40 sessions. We chose to conduct 40 sessions because this amount produced relatively stable responding. For example, the standard deviations of response rates across the last five sessions for which each component duration was presented averaged $15.5 \%$ of the mean response 
rate for the subjects exposed to the positive contrast procedure and $18 \%$ of the mean for the subjects exposed to the negative contrast procedure.

\section{RESULTS}

\section{Positive Contrast}

Figure 1 presents the mean rates of responding during the components of the baseline and contrast schedules for the subjects exposed to the positive contrast procedure. Each set of axes presents the results for a single component duration. Throughout this paper, the component in which the rate of reinforcement was held constant from the baseline to the contrast schedule will be labeled the constant component. The component in which the rate of reinforcement changed will be labeled the variable component. Responding in the constant component is indicated by the solid line; responding in the variable component is indicated by the dashed line.

Response rates were calculated by dividing the number of presses during a component by the time for which that component was available. The rates presented in Figure 1 are the means for all subjects responding over the last five sessions of exposure to each component duration. The results for the mean of all subjects represent those for individual subjects.

Figure 1 shows that behavior was under the control of the schedule of reinforcement. Response rates in the variable component consistently decreased when responding in that component was placed on extinction.

To determine whether contrast occurred, $t$ tests for matched pairs were applied to the rates of responding by individual subjects during the constant components of the baseline and contrast schedules for each component duration. Significant positive contrast occurred for the 30sec components $[t(4)=2.64, p=.03]$ and the 3-min components $[t(4)=8.88, p=.001]$. That is, the rates of responding during the constant component increased from the baseline to the contrast schedule. Contrast was also marginally significant for the 60-sec components [ $t(4)$ $=2.07, p=.055]$, but significant negative induction occurred for the 5-sec components $[t(4)=7.93, p=.001]$. Negative induction is defined as a decrease in the rate of responding during a constant component with decreases in the rate of reinforcement in the variable component. Neither contrast nor induction occurred for the 16-min components $[t(4)=0.12, p>.05]$.

Figure 2 presents the size of contrast plotted as a function of component duration in seconds for the median of all subjects. The size of contrast was calculated by dividing the rate of responding in the constant component during the contrast, multiple VI 15 -sec extinction schedule by the rate of responding in the same component during the baseline, multiple VI 15-sec VI 15-sec schedule for each subject and each component duration. The median of these ratios across all subjects is shown in Figure 2. Medians have been reported and nonparametric statistics will be used because these measures are ratios. The median results are representative of those for individual subjects. A logarithmic scale (base 10) has been used for component duration to separate the points for the shorter components. Contrast ratios that are greater than 1.0 represent positive contrast; points less than 1.0 represent negative induction.

A Friedman analysis of variance applied to the contrast ratios for individual subjects showed that the size of contrast changed significantly with component duration (Friedman test statistic $=15.00, p=.005$ ). Wilcoxon signed-ranks tests revealed that the ratio for the 5-sec component was significantly different from that for each of the other component durations $(p=.043)$. Ratios were also significantly different for the 30-sec and 3-min components and for the 3- and 16-min components $(p=.043)$.

The results presented in Figure 2 are ratios of rates of responding during the baseline and contrast schedules. Therefore, it may be asked how changes in each of these individual rates contributed to changes in the contrast ratios. A comparison of Figures 1 and 2 reveals that changes in the rates of responding during the constant components of the contrast schedules strongly influenced the contrast ratios. A one-way (component duration) within-subject analysis of variance showed that responding during the constant components of the contrast schedules changed significantly with component duration, as did the size of contrast $[F(4,16)=6.98, p<.01]$. The rates during the 5 -sec components differed from those during the $30-\mathrm{sec}$ $[t(4)=3.23, p=.03]$ and $60-\sec [t(4)=2.86, p=.05]$ components. The rates during the 30 -sec components differed from those during the $60-\mathrm{sec}$ components $[t(4)$ $=2.97, p=.04$ ].

Changes in the rates of responding during the constant components of the baseline schedules also contributed to the functions reported in Figure 2. Baseline rates of responding were lower for the 3-min components (41.1 responses per minute) than for the other components (mean $=63.0$ responses per minute), which contributed to the large size of contrast reported for the 3-min components. However, shifts in baseline response rates did not create the functions reported in Figure 2. A one-way within-subject analysis of variance showed that the rates during the constant components of the baseline schedules did not change significantly with changes in component duration $[F(4,16)=2.68, p>.05]$.

\section{Negative Contrast}

Figure 3 presents the mean rates of responding (presses per minute) during the variable (dashed line) and constant (solid line) components of the baseline and contrast schedules for the subjects exposed to the negative contrast procedure. Each set of axes presents the results for a single component duration.

Response rates were calculated and presented as for Figure 1, with the following exception. The data that entered the calculations of mean response rates were not those for the last five sessions for 3 subjects in the 5 -sec condition and 2 subjects in the 3- and 16-min conditions. These subjects responded slowly during the contrast 
$5-5$

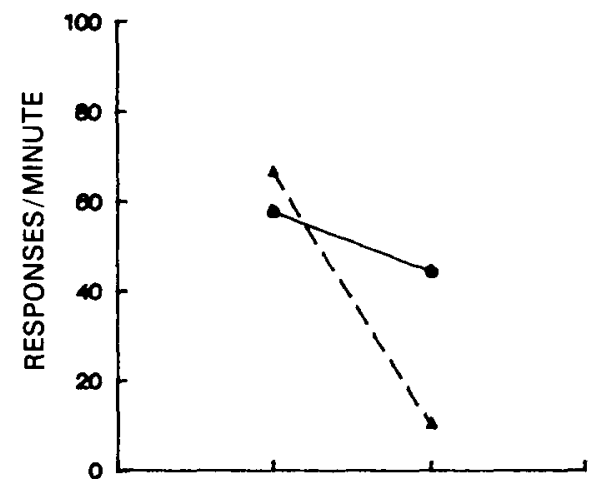

BASELINE CONTRAST

$30-5$

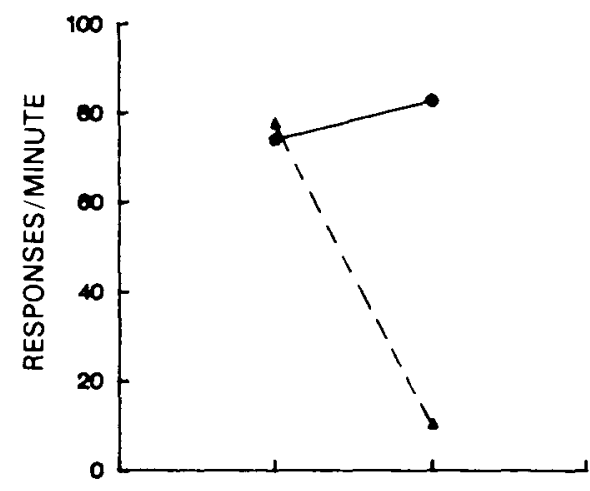

BASELINE CONTRAST

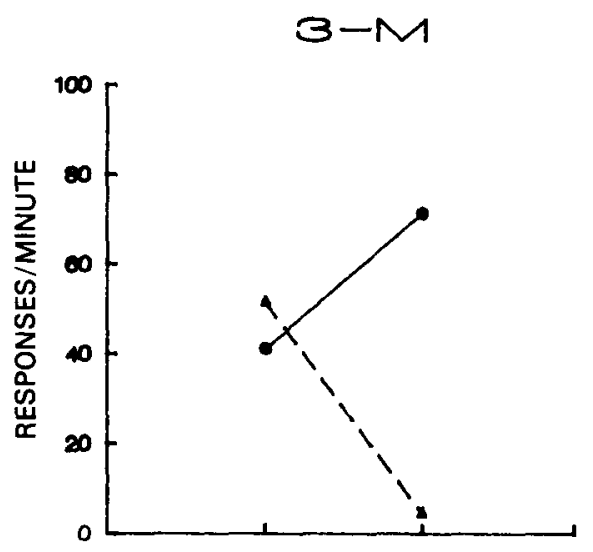

BASELINE CONTRAST

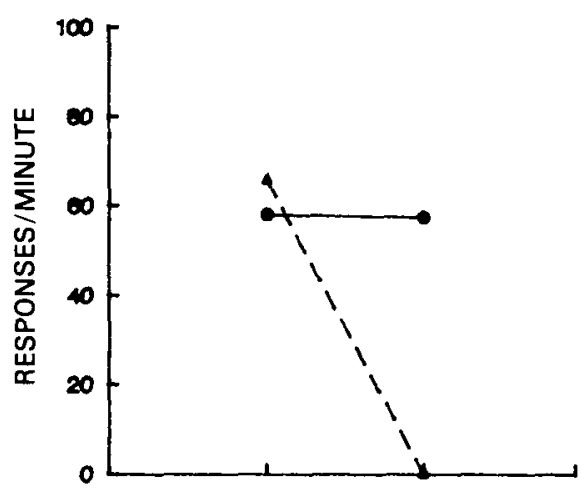

BASELINE CONTRAST

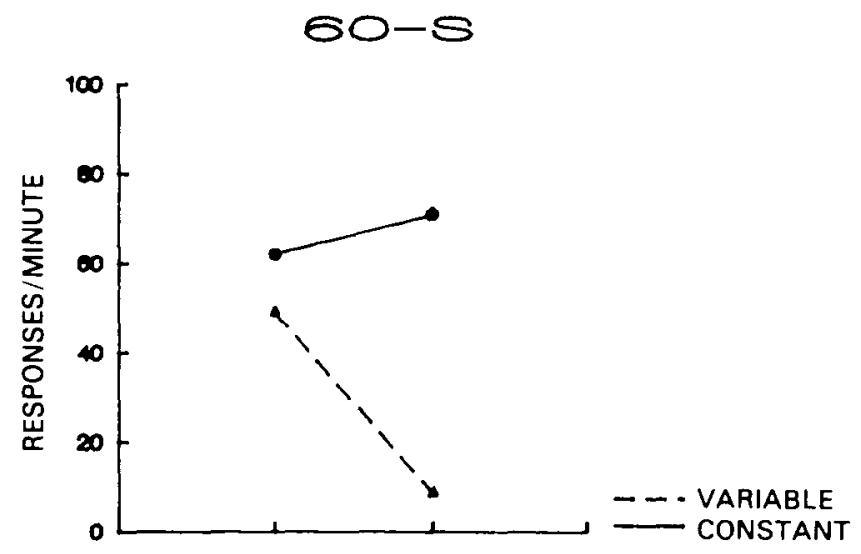

BASELINE CONTRAST

Figure 1. Mean rates of responding during the variable (dashed line) and constant (solid line) components of the baseline and contrast schedules for the subjects exposed to the positive contrast procedure. Each set of axes presents the results for a particular component duration. The rates are those for the mean of all subjects responding over the last five sessions for which each component duration was presented. 


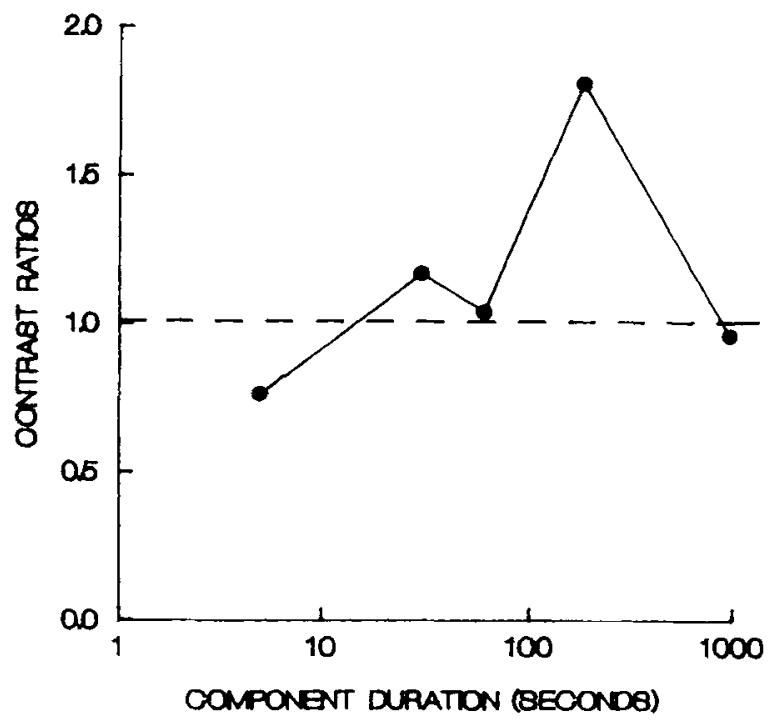

Figure 2. Positive contrast as a function of component duration in seconds. The size of contrast was measured by dividing the rate of responding on the lef lever of the multiple VI 15 -sec extinction schedule by the rate of responding on the left lever of the multiple VI 15-sec VI 15-sec schedule. Component duration has been plotted on a logarithmic (hase 10) scale. The results are those for the median of all subjects.

schedule in some sessions; as a result, their obtained rates of reinforcement fell. Contrast is not reported for those sessions because contrast is a change in responding that occurs even when the rate of reinforcement produced by that responding is constant. To avoid this problem, the last five sessions were sought during which the rate of reinforcement obtained in the constant component remained constant from the baseline to the contrast schedule. The data from those sessions entered the calculations of the mean response rates.

Figure 3 shows that the rates of responding during the two components of the baseline schedules were not equal, even though those components provided identical rates of reinforcement. These differences probably represent a preference for one operandum over another. Such differences frequently appear when the components of the multiple schedules are presented on two different operanda (e.g., Charman \& Davison, 1982).

Figure 3 also shows that the rates of responding during the variable component decreased from the baseline to the contrast schedules, even though the rate of reinforcement produced by that responding increased. Such decreases in response rates at very high rates of reinforcement are not unusual. They have been observed in other experiments (e.g., Dougan \& McSweeney, 1985) and are consistent with several theories (e.g., Baum, 1981; Staddon, 1979).

The decreases in response rates do not rule out the use of the present data to examine negative contrast. The conditions necessary for studying contrast are still present.
The rates of reinforcement obtained by responding during the variable component still increased substantially from the baseline (mean $=3.6$ reinforcers per minute) to the contrast schedules (mean $=8.4$ reinforcers per minute).

To determine whether negative contrast occurred, $t$ tests for matched pairs compared the rates of responding during the constant components of the baseline and contrast schedules for each component duration. Negative contrast occurred for all durations, except for the 5-sec components [for the 5 -sec components, $t(4)=1.35, p=.13$; for the 30 -sec components, $t(4)=3.87, p=.01$; for the 60 sec components, $t(4)=12.09, p=.000$; for the $3-\mathrm{min}$ components, $t(4)=5.52, p=.003$; for the 16 -min components, $t(4)=3.35, p=.02$ ]. That is, responding during the constant component decreased significantly from the baseline to the contrast schedules.

Figure 4 presents the size of negative contrast plotted as a function of the logarithm (base 10) of component duration in seconds for the median of all subjects. The size of contrast was determined by dividing the rate of responding in the constant component of the contrast, multiple VI 15-sec VI 7-sec schedule by the rate of responding in the constant component of the baseline, multiple VI 15sec VI 15-sec schedule. Again, medians and nonparametric statistics have been reported because these points are ratio measures. The results for the median of all subjects basically represent those for individual subjects. Points that fall below 1.0 represent negative contrast.

A Friedman analysis of variance applied to the contrast ratios for individual subjects showed that the size of negative contrast changed significantly with component duration (Friedman test statistic $=14.76, p=.005$ ). Wilcoxon signed-rank tests revealed that the contrast ratio for the 5-sec component was significantly different from the ratio for each of the other component durations $(p=$ .043). The ratio for the 3-min component was also significantly different from that for the 16-min component $(p=.043)$.

A comparison of Figures 3 and 4 shows that changes in responding during the constant components of the contrast schedules contributed strongly to the functions reported in Figure 4. A one-way (component duration) within-subject analysis of variance showed that these response rates changed significantly with component duration $[F(4,16)=17.54, p<.0001]$. Response rates during the 5-sec component differed from those during the $30-\sec [t(4)=9.32, p<.002], 60-\sec [t(4)=7.17$, $p<.01]$, and 3-min $[t(4)=6.19, p<.01]$ components. Response rates also differed for the 30-sec and 16-min $[t(4)=3.05, p<.05]$ components and for the 3 - and $16-\min [t(4)=3.26, p<.05]$ components. These absolute rates of responding were highly correlated with the sizes of contrast reported in Figure $4(r=0.90$, for the mean of all subjects).

Changes in responding during the constant components of the baseline schedules also contributed to the functions reported in Figure 4, but less strongly. These rates 


$$
5-S
$$

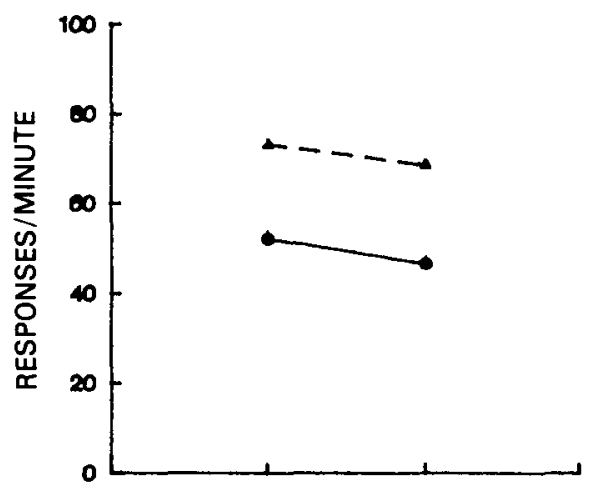

BASELINE CONTRAST
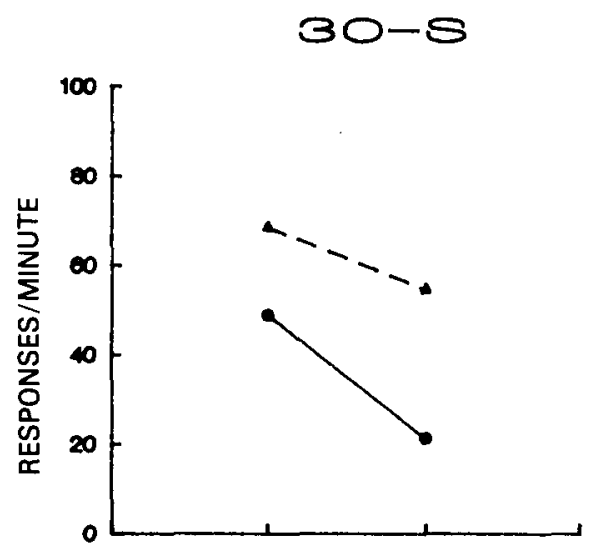

BASELINE CONTRAST

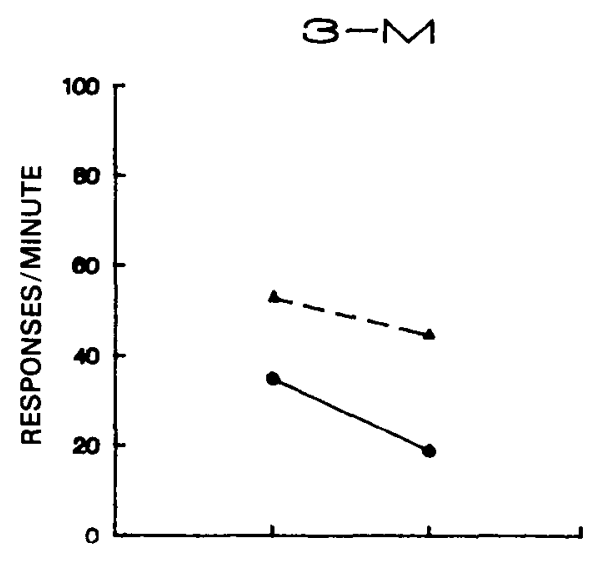

BASELINE CONTRAST

$76-M$

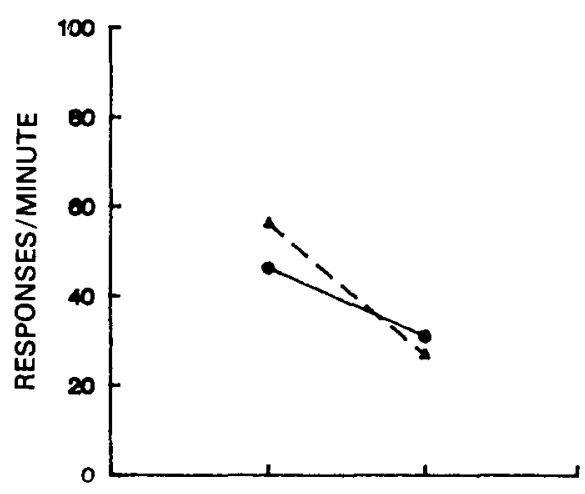

BASELINE CONTRAST
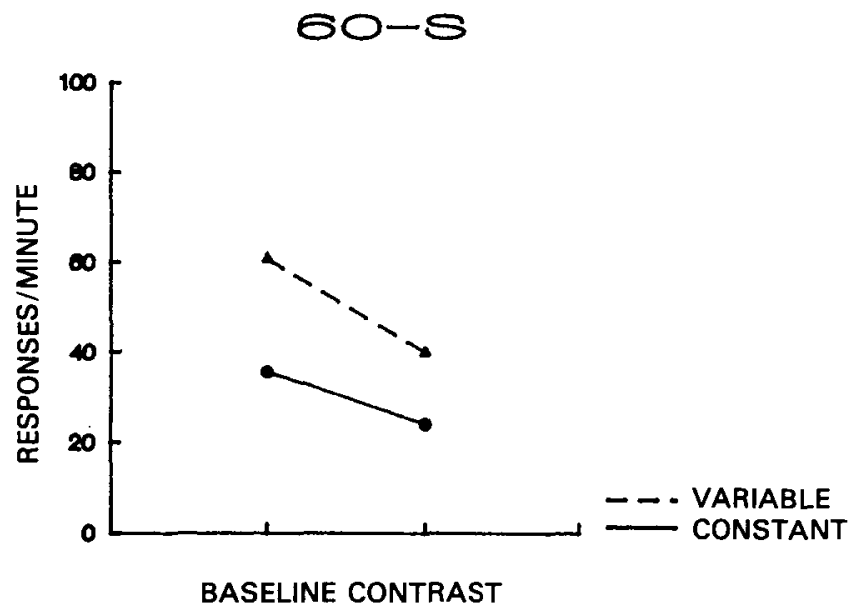

Figure 3. Mean rates of responding during the variable (dashed line) and constant (solid line) components of the baseline and contrast schedules for the subjects exposed to the negative contrast procedure. Each set of axes presents the results for a particular component duration. The rates are those for the mean of all subjects responding over the last five sessions for which each component duration was presented. 


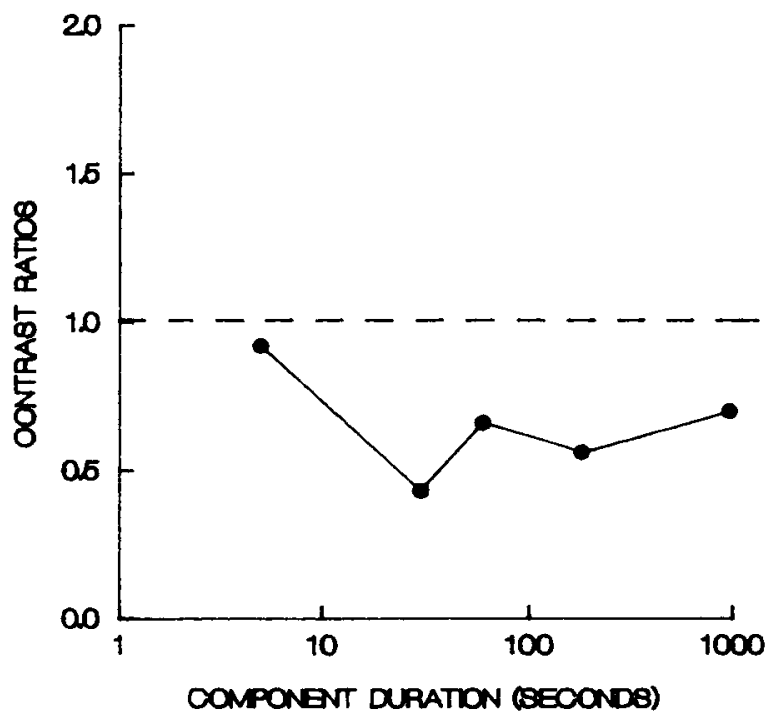

Figure 4. Negative contrast as a function of component duration in seconds. The size of contrast was measured by dividing the rate of responding on the left lever of the multiple VI 15-sec VI 7-sec schedule by the rate of responding on the left lever of the multiple VI 15-sec VI 15-sec schedule. Component duration has been plotted on a logarithmic (base 10) scale. The results are those for the median of all subjects.

changed significantly with component duration $[F(4,16)$ $=3.62, p<.05]$. Response rates during the 5 - $\mathrm{sec}$ components differed from those during the $60-\sec [t(4)=$ $3.88, p<.02]$ and $3-\min [t(4)=7.17, p<.01]$ components. However, these rates were not very highly correlated with the reported contrast ratios $(r=0.32$, for the mean of all subjects).

\section{DISCUSSION}

\section{Leverpress Contrast and Component Duration}

Figure 2 shows that the size of positive contrast increased with increases in component duration up to $3 \mathrm{~min}$ and then decreased again. Negative induction occurred for the 5-sec components. Figure 4 shows that the absolute size of negative contrast increased with increases in component durations up to $30 \mathrm{sec}$ and then decreased with further increases. These results have at least three implications.

First, behavioral contrast occurred for leverpressing and was sometimes large. Response rates changed by more than $50 \%$ between the baseline and contrast schedules when appropriate parameters were chosen. This contradicts the conclusion that contrast does not occur when rats press levers for food reinforcers (e.g., Rachlin, 1973). Second, the present results may help to explain some of the past failures to observe positive contrast with the leverpress response. Positive contrast was large in the present experiment, but only when the components were $3 \mathrm{~min}$ long. Third, the present results question theories that predict that the size of contrast varies inversely with component duration (e.g., Herrnstein, 1970; Rachlin, 1973). Instead, the absolute sizes of both positive and negative contrast increased with initial increases in component duration and then decreased. Because of the importance of these functions, several possible explanations for them will be considered.

First, changes in discrimination across component durations might have produced these functions. Good discrimination is required to produce contrast (e.g., Rachlin, 1973). Therefore, changes in the quality of the discrimination might produce changes in the size of contrast. To test this idea, a discrimination ratio was calculated for each contrast schedule. The rate of responding during the constant component was divided by the sum of the rates of responding during both components. As predicted, these discrimination ratios were correlated with the sizes of contrast for the mean of all subjects (positive contrast, $r=0.50$; negative contrast, $r=0.56$ ). Changes in discrimination were not, however, necessary to produce these functions for individual subjects. The correlation between the discrimination ratio and the size of contrast was negative or zero for 1 subject in each experiment, but the functions reported for those subjects were similar to the functions reported for the other subjects.

The number of components presented per session was confounded with component duration in the present study. This was done to make the present procedure comparable to that used in past studies. However, Williams (1983) has argued that the following component plays a larger role in the production of contrast than does the preceding component. If this is true, then more contrast might be found when more components, and therefore more following components, are presented. If the number of following components determined the size of contrast, then contrast should have been larger for shorter components than for longer ones. Instead, contrast was small for the 5 -sec components, and it increased with increases in component duration up to a point.

The preceding argument could be modified to state that the amount of time spent in the following component, not the number of appearances of that component, affects the size of contrast. Then, contrast should be largest for the 16-min components, smaller for the 3-min components, and smallest for the 5-, 30-, and 60-sec components. The obtained functions also differ substantially from this prediction.

The proportion of the total session time that was spent in a timeout was also confounded with component duration. Three-second timeouts separated all components. More timeouts occurred for the shorter components than for the longer components because more shorter components were presented per session. Changes in the number of timeouts might alter response rates by changing the perceived rates of reinforcement. No reinforcers were delivered during the timeouts. Therefore, perceived rates of reinforcement would be lower for the shorter components than for the longer components if subjects included 
timeouts in calculating rates of reinforcement. Lower perceived rates of reinforcement should produce lower rates of responding. However, baseline response rates did not change significantly with component duration for positive contrast. They did for negative contrast, but response rates were highest for the 5-sec components and lowest for the 3-min components, opposing the prediction.

The present results might be by-products of changes in the number of incorrect responses with changes in component duration. The left lever remained available when the right component was in effect, and vice versa. Therefore, responses on the incorrect lever may have competed with responses on the correct lever to produce the present functions. This hypothesis can also be rejected. Very few incorrect responses were emitted in either experiment. For example, the rate of incorrect responding during the constant component of the positive contrast schedule varied from a mean of 0.1 to a mean of 0.7 responses per minute for different component durations. Rates of incorrect responding also failed to change significantly with component duration for either positive $[F(4,16)=2.71$, $p>.05]$ or negative $[F(4,16)=2.40, p>.05]$ contrast.

Levels of satiation may also have been confounded with component duration. Session lengths were selected to be similar to those used in the past studies. This produced 40-min sessions (excluding time spent in timeouts) for the 5-, 30-, and 60-sec components, 48-min sessions for the 3-min component, and 64-min sessions for the 16-min component. Differences in session length may have produced different levels of satiation because high rates of reinforcement were used. It does not seem likely that satiation played a role during the baseline schedules. If it had, baseline response rates would have been lower for longer sessions. Baseline response rates did not vary with component duration for positive contrast and did not fit the pattern predicted by satiation for negative contrast. For example, differences in response rates would be expected between the shortest and longest sessions, but $t$ tests for matched pairs showed that the rates of responding during the constant components were not significantly different for the 5-sec and 16-min durations $[t(4)=0.84$, $p>.05]$ or for the $30-\mathrm{sec}$ and 16-min durations $[t(4)=$ $0.51, p>.05$ ].

On the other hand, satiation may have played a role during the contrast schedules for negative contrast during the 16-min condition. The multiple VI 15-sec VI 7-sec schedule provided the highest rates of reinforcement; sessions were longest for the 16-min components. Therefore, satiation might be expected. As predicted, response rates during the VI 7-sec components were significantly lower for the 16-min components than for all other component durations [for the 5-sec components, $t(4)=4.00$, $p<.01$; for the 30 -sec components, $t(4)=4.46$, $p<.01$; for the 60 -sec components, $t(4)=2.51$, $p<.05$; for the 3-min components, $t(4)=4.59$, $p<.01]$.

However, response rates during the VI 7-sec components do not fit the pattern predicted by satiation for the other component durations. The 5-, 30-, and 60-sec com- ponents were available for the same amount of time and should have produced similar amounts of satiation, but the rates of responding during those components differed significantly [5-sec vs. 30-sec components, $t(4)=2.20$, $p<.05 ; 5$-sec vs. 60 -sec components, $t(4)=3.60$, $p<.025 ; 30$-sec vs. 60 -sec components, $t(4)=5.87$, $p<.005]$. Likewise, response rates did not differ for components that appeared for different amounts of time and should have produced different amounts of satiation [30-sec vs. 3-min components, $t(4)=1.61, p>.05$; 60 -sec vs. 3 -min components, $t(4)=0.73, p>.05$ ]

Finally, as in past studies, cue color and lever location were confounded with whether the lever was associated with the constant or the variable component. Differences between these cues or levers may have distorted the measures of the absolute size of contrast, leading to an overor underestimate of its absolute size in the present experiments. However, past data suggest that such differences do not distort the basic form of the function relating the size of contrast to component duration. If such differences distorted the functions, the results of past studies that have used two different operanda to present the components should differ from those of studies that have used only one. Instead, the function relating the size of keypeck contrast to component duration is virtually identical regardless of whether a one-operandum or two-operanda procedure is used (compare the results of McSweeney, 1982, to those of McSweeney \& Melville, 1988).

In sum, changes in discrimination may have contributed to the forms of the present functions. Satiation may have also contributed to the lower response rates during the 16-min components in the contrast phase for negative contrast. But, for the most part, the factors that produced the present functions are unknown, as they are for other responses, such as keypecking and treadlepressing (e.g., McSweeney, 1982). Future experiments should explore alternative explanations for the form of these functions.

\section{Symmetry of Positive and Negative Contrast}

The results presented in Figures 2 and 4 generally support theories that attribute positive and negative contrast to similar theoretical mechanisms (e.g., Herrnstein, 1970; Hinson \& Staddon, 1978; Rachlin, 1973). The absolute sizes of both positive and negative contrast were smallest for the shortest components and increased with increases in component duration up to a point. However, some differences also appeared. The greatest contrast was found for the 3-min components for positive contrast and for the 30-sec components for negative contrast. Contrast ratios for the $30-\mathrm{sec}$ components differed from those for the 3-min components for positive but not for negative contrast. Negative induction also occurred instead of positive contrast for the 5-sec components, but positive induction did not occur instead of negative contrast.

The factors that produced these differences should be determined. They may have important theoretical implications, or they may be procedural by-products. Although the procedures used to measure positive and negative contrast were as similar as possible, one difference may al- 
ways occur. Until a scale of reinforcer value is developed, it cannot be assumed that the size of the decrease in the rate of reinforcement that is used to produce positive contrast is equal to the size of the increase in the rate of reinforcement that is used to produce negative contrast. Therefore, quantitative differences between the two types of contrast may always be attributed to quantitative differences in the manipulations used to produce them.

\section{Contrast for Different Responses}

Figure 5 compares the present functions for leverpressing to similar functions for keypecking and treadlepressing. Contrast has been measured as in Figures 2 and 4 and has been plotted as a function of the logarithm (base 10) of the component duration in seconds. The axes on the top present positive contrast; those on the bottom present negative contrast. The data for keypecking are represented by circles, those for leverpressing by triangles, and those for treadlepressing by squares. The results for positive keypeck contrast were taken from McSweeney and Melville (1988), those for negative keypeck and treadlepress contrast from McSweeney (1982), and those for positive treadlepress contrast from McSweeney et al. (1986). All of the results are those for the median of all subjects responding in each experiment.

Figure 5 shows that both positive and negative contrast changed as similar functions of component duration for leverpressing and treadlepressing. The functions for these responses differed from those for keypecking. Positive keypeck contrast generally decreased with increases in component duration. Positive leverpress or treadlepress contrast increased and then decreased with increases in component duration. The absolute size of negative keypeck contrast decreased with initial increases in component duration and then changed erratically with further changes in component duration. Negative leverpress or treadlepress contrast increased with initial increases in component duration and then changed erratically.

These differences between responses were produced mainly by different changes in absolute response rates during the constant components of the contrast schedules. For positive contrast, the response rates during the constant components of the baseline schedules did not change significantly with component duration for keypecking $[F(4,8)$ $=0.494, p>.05]$, for leverpressing $[F(4,16)=2.68$, $p>.05]$, or for treadlepressing $[F(3,6)=0.649$, $p>.05]$. Instead, different changes in the rates of responding during the constant components of the contrast schedules determined the different forms of the functions.

For negative contrast, response rates during the constant components of the baseline schedules did change significantly with component duration for all responses [for keypecking, $F(3,9)=13.957, p=.001$; for leverpressing, $F(4,16)=17.54, p<.0001$; for treadlepressing, $F(4,12)=11.751, p=.000]$. However, the function

\section{POSTTNE CONTRAST}

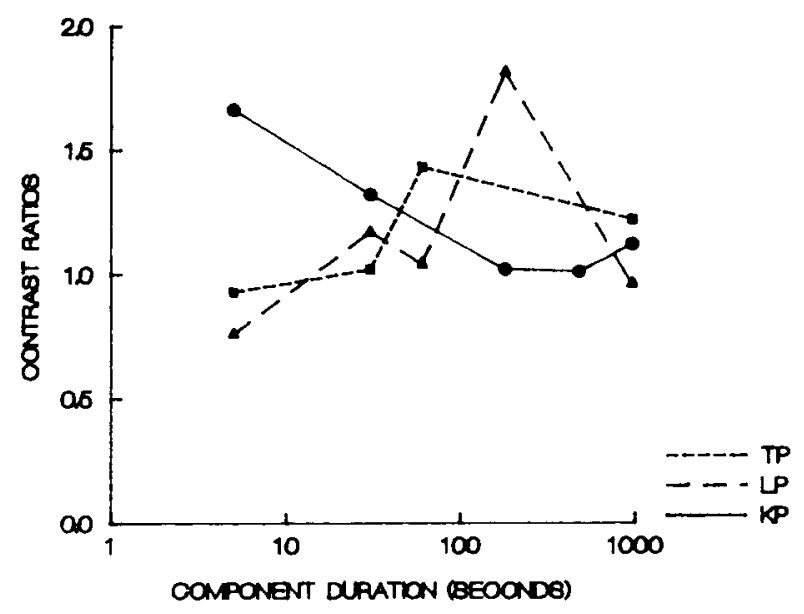

NEGATIVE CONTRAST

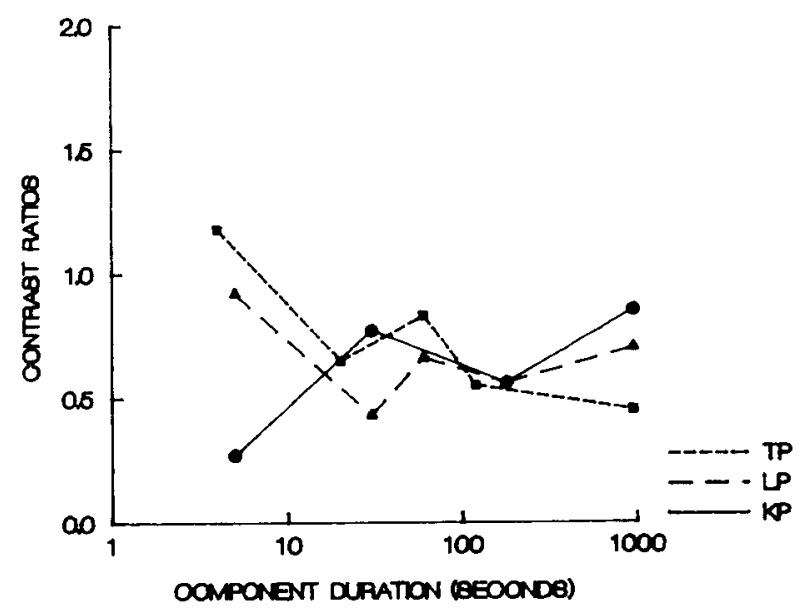

Figure 5. Contrast ratios plotted as a function of component duration in seconds. The top axis presents positive contrast; the bottom presents negative contrast. Results for keypecking are represented by circles, those for leverpressing by triangles, and those for treadlepressing by squares. In all cases, contrast was measured by dividing the rate of responding during the constant component of the contrast schedule by the rate of responding during the constant component of the baseline schedule. The results for leverpressing were taken from the present study, those for positive keypeck contrast from McSweeney and Melville (1988), those for negative keypeck and treadlepress contrast from McSweeney (1982), and those for positive treadiepress contrast from McSweeney et al. (1986). Component duration has been plotted on a logarithmic (base 10) scale. All data are those for the median of all subjects. 
reported in Figure 5 for keypecking was determined mainly by a large increase in response rates during the constant component of the contrast schedules from the shortest to the next shortest component duration (mean $=13.0$ to 41.5 responses per minute). This change was opposite to the large decrease in response rates from the shortest to the next shortest components for both leverpressing (mean $=46.6$ to 21.4 responses per minute) and treadlepressing (mean $=18.4$ to 9.9 responses per minute).

The factors that produced the different functions for keypecking and leverpressing or treadlepressing should be determined. Most obvious procedural factors can be ruled out because the procedures used to measure positive keypeck and leverpress contrast were similar in most obvious ways. Because the studies were conducted in the same laboratories, less obvious factors (e.g., the handling of the subjects, the time of day at which the studies were conducted) were also similar.

All attempts to compare across responses are inherently flawed. Procedures may differ in important, but as yet unidentified, ways even when as many factors as possible have been equated across responses. The present experiment has, however, taken the best approach currently available. This approach is similar to the functional approach advocated by Bitterman $(1960,1965)$ for comparing across species. As argued earlier, this functional approach is relatively strong because it rules out many of the simplest explanations for differences in results across responses or species.

If it is accepted that the results presented in Figure 5 represent a reasonable comparison of responses, then they have at least two implications. First, they suggest that keypecking and leverpressing change in different ways when subjected to procedures that are very similar. On the surface, this evidence provides a strong challenge to all of the general theories of contrast (e.g., Herrnstein, 1970; Hinson \& Staddon, 1978; McSweeney, 1987; Williams, 1983).

Second, the results help to clarify how the general theories fail. Because relatively similar results were found for leverpressing by rats and treadlepressing by pigeons, species differences do not appear to be critical to obtaining different functions. Because different results were found for keypecking and leverpressing, factors related to response topography do appear to be critical. Future experiments should identify these factors.

\section{REFERENCES}

BAUM, W. M. (1981). Optimization and the matching law as accounts of instrumental behavior. Journal of the Experimental Analysis of Behavior, 36, 387-403.

Bitterman, M. E. (1960). Toward a comparative psychology of learning. American Psychologist, 15, 704-712.

Bitterman, M. E. (1965). Phyletic differences in leaming. American Psychologist, 20, 396-410.

Charman, L., Davison, M. (1982). On the effects of component durations and component reinforcement rates in multiple schedules. Joumal of the Experimental Analysis of Behavior, 37, 417-439.
CoElho DE Rose, J. C. (1986). Behavioral contrast in fixed-interval components: Effects of extinction-component duration. Journal of the Experimental Analysis of Behavior, 45, 175-188.

Davison, M., \& Ferguson, A. (1978). The effects of different component response requirements in multiple and concurrent schedules. Journal of the Experimental Analysis of Behavior, 29, 283-295.

Dougan, J. D., \& McSweeney, F. K. (1985). Variations in Herrnstein's ro as a function of alternative reinforcement rate. Journal of the Experimental Analysis of Behavior, 43, 215-223.

Dougan, J. D., McSweeney, F. K., Farmer, V. A. (1985). Some parameters of behavioral contrast and allocation of interim behavior in rats. Journal of the Experimental Analysis of Behavior, 44, 325-335.

Fleshler, M., \&offMAN, H. S. (1962). A progression for generating variable-interval schedules. Joumal of the Experimental Analysis of Behavior, 5, 529-530.

GAMZU, E., \& SCHWARTZ, B. (1973). The maintenance of key pecking by stimulus-contingent and response-independent food presentation. Journal of the Experimental Analysis of Behavior, 19, 65-72.

Hearst, E., \& JenKINS, H. M. (1974). Sign-tracking; The stimulusreinforcer relation and directed action. Austin, TX: Psychonomic Society.

Hemmes, N. S. (1973). Behavioral contrast in pigeons depends upon the operant. Journal of Comparative \& Physiological Psychology, 85, 171-178.

HerRnstein, R. J. (1970). On the law of effect. Joumal of the Experimental Analysis of Behavior, 13, 243-266.

Hinson, J. M., \& StADDon, J. E. R. (1978). Behavioral competition: A mechanism for schedule interactions. Science, 202, 432-434.

MCSWEENEY, F. K. (1978). Negative behavioral contrast on multiple treadle-press schedules. Journal of the Experimental Analysis of Behavior, 29, 463-473.

MCSWEENEY, F. K. (1982). Positive and negative contrast as a function of component duration for key pecking and treadle pressing. Journal of the Experimental Analysis of Behavior, 37, 281-293.

MCSWEENEY, F. K. (1987). Suppression by reinforcement, a model for multiple-schedule behavioral contrast. Behavioural Processes, 15, 191-209.

McSweeney, F. K., Dougan, J. D., Higa, J., \& Farmer, V. A. (1986). Behavioral contrast as a function of component duration and baseline rate of reinforcement. Animal Learning \& Behavior, 14, 173-183.

MCSweeney, F. K., \& Melville, C. L. (1988). Positive contrast as a function of component duration using a within-session procedure. Behavioural Processes, 16, 21-41.

Rachlin, H. (1973). Contrast matching. Psychological Review, 80, 217-234.

SCHWARTZ, B. (1975). Discriminative stimulus location as a determinant of positive and negative behavioral contrast in the pigeon. Journal of the Experimental Analysis of Behavior, 23, 167-176.

Seligman, M. E. P. (1970). On the generality of the laws of learning. Psychological Review, 77, 406-418.

Spealman, R. D., \& Gollub, L. R. (1974). Behavioral interactions in multiple variable-interval schedules. Joumal of the Experimental Analysis of Behavior, 22, 471-481.

STADDON, J. E. R. (1979). Operant behavior as adaptation to constraint. Journal of Experimental Psychology: General, 108, 48-67.

STADDON, J. E. R. (1982). Behavioral competition, contrast and matching. In M. L. Commons, R. J. Hermstein, \& H. Rachlin (Eds.), Quantitative analyses of behavior, matching and maximizing accounts (Vol. 2, pp. 243-262). Cambridge, MA: Ballinger.

WESTBRook, R. F. (1973). Failure to obtain positive contrast when pigeons press a bar. Joumal of the Experimental Analysis of Behavior, 20, 499-510.

Williams, B. A. (1979). Contrast, component duration, and the following schedule of reinforcement. Journal of Experimental Psychology: Animal Behavior Processes, 5, 379-396.

Williams, B. A. (1983). Another look at contrast in multiple schedules. Journal of the Experimental Analysis of Behavior, 39, 345-384.

(Manuscript received May 19, 1989;

revision accepted for publication August 14, 1990.) 